\title{
Review of: "The atlas of human cardiac promoters and enhancers reveals an important role for regulatory elements in heart failure"
}

\author{
Mun-Kit Choy ${ }^{1}$ \\ 1 The University of Manchester
}

Potential competing interests: The author(s) declared that no potential competing interests exist.

Congratulations on completing an atlas of regulatory elements in heart failure by conducting a CAGE project. This is a valuable resource for cardiovascular scientists, especially those who are interested in identifying target genes to understand or even to cure the cardiac condition. You have also performed comprehensive analyses to put the atlas in perspective, so a job well done. The manuscript has been written in a descriptive way which is helpful for readers to explore this resource. I would however prefer to have some sort of statistical framework to provide "confidence levels" or "weights" when reading through the conclusions. For example I didn't get how representative the heart clusters are among the 21 healthy or 10 failing hearts. Also some of the conclusions seem to have been made through specific examples. For instance, when investigating the TSS usage only several genes strongly associated with heart failure were selected. In the case of GWAS analysis, I didn't get how many GWAS studies were included in the "heart GWAS" and what was the statistical significance of the "overlap" or "enrichment" between the GWAS hits and CAGE clusters. The manuscript indicates that one of the genes, TNNI3, is linked with an SNP. Is it just because they are close to each other? Or are there more evidence such as eQTLs or chromosomal interactions? Perhaps eQTLs or chromosome interactions could help to define the relationship between promoters and enhancers identified in this study? In addition, some wet lab verification on the CAGE clusters or conclusions that the authors are highlighting would also give the readers more confidence. 